\title{
ABSOLUTE TWO PLASMON PARAMETRIC DECAY \\ $O F$ \\ OBLIQUELY INCIDENT ELECTROMAGNETIC WAVES \\ IN AN INHOMOGENEOUS PLASMA
}

BY

J. J, SCHUSS

\section{PLASMA PHYSICS LABORATORY}

\section{MASTER}

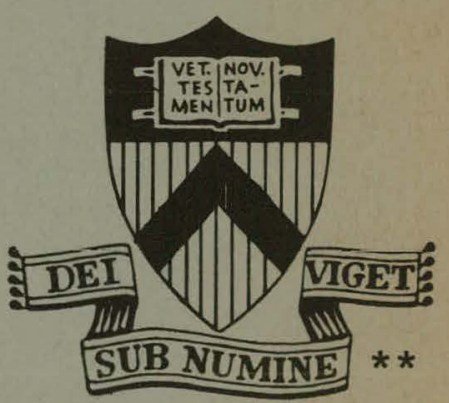

\section{PRINCETON UNIVERSITY PRINCETON, NEW JERSEY}

This work was supported by U. S. Energy Research and Development Administration Contract $\mathrm{E}(11-1)-3073$. Reproduction, translation, publication, use and disposal, in whole or in part, by or for the United States Government is permitted. 


\section{DISCLAIMER}

This report was prepared as an account of work sponsored by an agency of the United States Government. Neither the United States Government nor any agency Thereof, nor any of their employees, makes any warranty, express or implied, or assumes any legal liability or responsibility for the accuracy, completeness, or usefulness of any information, apparatus, product, or process disclosed, or represents that its use would not infringe privately owned rights. Reference herein to any specific commercial product, process, or service by trade name, trademark, manufacturer, or otherwise does not necessarily constitute or imply its endorsement, recommendation, or favoring by the United States Government or any agency thereof. The views and opinions of authors expressed herein do not necessarily state or reflect those of the United States Government or any agency thereof. 


\section{DISCLAIMER}

Portions of this document may be illegible in electronic image products. Images are produced from the best available original document. 
This report was prepared as an account of work sponsored by the United States Government. Neither the United States nor the United States Energy Research and Development Administration, nor any of their employees, nor any of their contractors, subcontractors, or their employees, makes any warranty, express or implied, or assumes any legal liability or responsibility for the accuracy, completeness or usefulness of any information, apparatus, product or process disclosed, or represents that its use would not infringe privately owned rights.

Printed in the United States of America.

Available from

National Technical Information Service

U. S. Department of Commerce 5285 Port Royal Road

Springfield, Virginia 22151

Price: Printed Copy \$__; Microfiche $\$ 1.45$

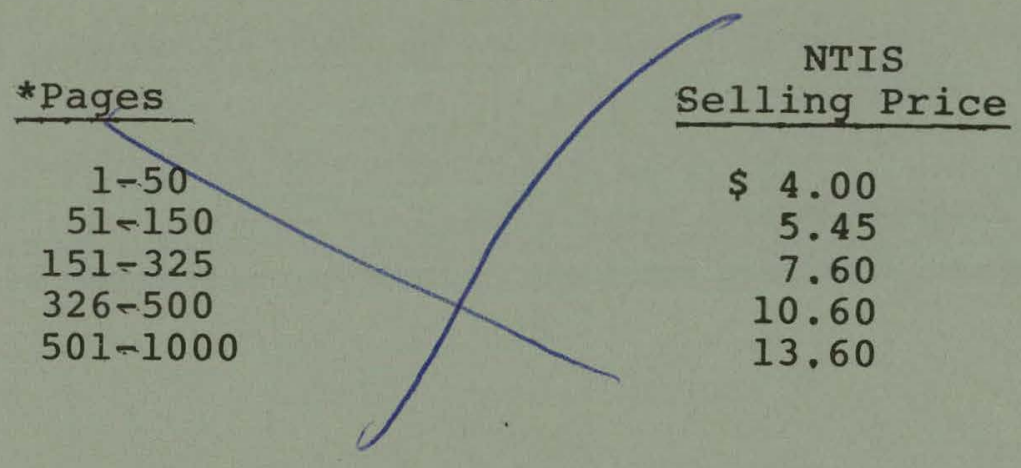


Absolute Two Plasmon Parametric Decay of Obliquely Incident Electromagnetic Waves in an Inhomogeneous Plasma

\author{
J. J. Schuss \\ Princeton University, Plasma Physics Laboratory \\ Princeton, New Jersey 08540, USA
}




\author{
ABSOLUTE TWO PLASMON PARAMETRIC DECAY \\ OF OBLIQUELY INCIDENT ELECTROMAGNETIC WAVES \\ IN AN INHOMOGENEOUS PLASMA
}

\author{
J. J. Schuss \\ Plasma Physics Laboratory, Princeton University \\ Princeton, New Jersey 08540
}

\begin{abstract}
The parametric decay of an electromagnetic wave into two electron plasma waves is considered. The electromagnetic wave is obliquely incident on the density gradient. The resulting analysis shows that the threshold for absolute instability decreases as the angle of incidence of the electromagnetic wave relative to the density gradient approaches $90^{\circ}$. The importance of this result to laser fusion is discussed.
\end{abstract}


The parametric decay of electromagnetic waves of frequency and wave number $\left(\omega_{0}, \vec{k}_{0}\right)$ into plasma waves $(\omega, \vec{k})$ at quartercritical density in an inhomogeneous plasma has been extensively discussed in the literature. Rosenbluth ${ }^{1}$ pointed out that in the WKB approximation this degcay process is convective in nature, and requires a minimum gradient length $L>\left[k_{0}\left(v_{o} / v_{e}\right)^{2}\right]^{-1}$ for the decay waves to grow to an appreciable level; here, $v_{0}$ is the electron velocity due to the pump, and $v_{\rho}^{2}=T_{\rho} / m_{\rho}$. Lee and Kaw ${ }^{2}$ studied the relevant equations without using any WKB approximation for the plasma waves and found that this decay can be absolute; this would permit the decay waves to grow until their amplitude is limited by nonlinear saturation effects. However, Lee and Kaw did not include the wave number mismatch introduced by the pump field into the parametric coupling terms. It is precisely this term which forbids an absolute instability. in Rosenbluth's paper. Liu and Rosenbluth ${ }^{3}$ re-examined this decay and found that when the wave number mismatch was included, absolute instability could exist for $\left|v_{0}\right|>\frac{3}{2} k_{\perp} v_{e}^{2} / \omega_{p}$, where $k_{\perp}$ is the component of the plasmon wave vector perpendicular to the density gradient, and $\omega_{p}$ is the plasma frequency.

In this article the relevant equations for the two plasmon decay are studied for a pump wave obliquely incident on the density gradient. This reduces but does not eliminate the mismatch term, making an absolute instability easier to generate. No WK̈B assumption is made concerning the plasma waves. The pump field $\overrightarrow{\mathrm{E}}_{0}(\overrightarrow{\mathrm{x}}, t)=\overrightarrow{\mathrm{E}}_{0} / 2\left[\exp \left(i \overrightarrow{\mathrm{k}}_{0} \cdot \overrightarrow{\mathrm{x}}-i \omega_{0} t\right)+\mathrm{c} . c.\right]$ is assumed to vary slowly in the region of the instability. For this to be 
true, the pump cutoff point must be far from the quarter critical density point. ${ }^{4}$ It can easily be shown that this is equivalent to requiring $\sin ^{2} \theta>\frac{1}{3}\left(3 / k_{0} L\right)^{2 / 3}$, where $(\pi / 2-\theta)$ is the angle between the density gradient and $\vec{k}_{0}$ at the quarter critical density point, and $L$ is the density scale length. We assume $\overrightarrow{\mathrm{E}}_{\mathrm{O}}=\mathrm{E}_{\mathrm{O}}\left[\alpha \hat{\mathrm{y}}+\left(1-\alpha^{2}\right)^{1 / 2}(\hat{z} \cos \theta-\hat{\mathbf{x}} \sin \theta)\right]$ and $\overrightarrow{\mathrm{k}}_{0}=\mathrm{k}_{0}(\hat{z} \sin \theta+\hat{\mathrm{x}} \cos \theta)(\alpha \leq 1)$. There are two decay waves present, $\phi(\bar{x}, t)=\phi(z) \exp (i \vec{k} \cdot \vec{x}-i \omega t)$ and $\phi_{-}(\bar{x}, t)=\phi_{-}(z) \cdot$ $\exp \left[i\left(\overrightarrow{\mathrm{k}}-\overrightarrow{\mathrm{k}}_{0}\right) \cdot \overrightarrow{\mathrm{x}}-i\left(\omega-\omega_{0}\right) t\right]$, and $\omega \approx \omega_{0} / 2$. Using the fluid equations, we arrive at an equation similar to that of Lee and Kaw :

$$
\begin{gathered}
\nabla \cdot\left[\frac{3 v_{e}^{2}}{\omega^{2}} \nabla \nabla \cdot+1-\frac{\omega_{\mathrm{p}}^{2}}{\omega^{2}}+\frac{2 \gamma}{\omega}\right] \nabla \phi \\
=\frac{e}{m \omega_{0}^{2}} \nabla \cdot\left[\nabla\left(\vec{E}_{0} \cdot \nabla \phi_{-}\right)-\nabla^{2} \phi_{-} \vec{E}_{0}\right] \\
\nabla \cdot\left[\frac{3 v_{e}^{2}}{\left(\omega-\omega_{o}\right)^{2}} \nabla \nabla \cdot+1-\frac{\omega_{p}^{2}}{\left(\omega-\omega_{0}\right)^{2}}-\frac{2 \gamma}{\omega}\right] \nabla \phi_{-} \\
=\frac{e}{m \omega_{0}^{2}} \nabla \cdot\left[\nabla\left(\vec{E}_{0} \cdot \nabla \phi\right)-\nabla^{2} \vec{E}_{0}\right]
\end{gathered}
$$

Here, $\gamma=\Delta+i r, \omega=\omega_{r}+\Delta, \omega_{r}$ is the plasmon frequency in the absence of a pump, $\Gamma$ is decay constant of the plasma waves, and $|\Delta| \ll\left|\omega_{r}\right|$. We now let $\vec{k}=k_{x} \hat{x}+k_{y} \hat{y}=\vec{k}_{\perp}$, $\vec{k}_{\perp_{-}}=\left(k_{x}-k_{o x}\right) \hat{x}+k_{y} \hat{y}$, where $k_{o x}=k_{o} \cos \theta$, and $\omega_{p}^{2}=$ $\left(w^{2}-3 v_{e}^{2} k_{\perp}^{2}\right)(1+z / L)$. We then obtain

$$
\begin{aligned}
& \left(\frac{3 v_{e}^{2}}{\omega^{2}} \frac{\partial^{2}}{\partial z^{2}}-\frac{z}{L}+\frac{2 \gamma}{\omega}\right)\left(\frac{\partial^{2}}{\partial z^{2}}-k_{\perp}^{2}\right) \phi-\frac{1}{L} \frac{\partial \phi}{\partial z} \\
& \quad=\frac{\alpha i e k_{y} E_{o}}{m \omega_{o}^{2}} \exp \left(i k_{o z} z\right)\left(2 i k_{o z} \frac{\partial \phi-}{\partial z}-2 k_{x} k_{o x}{ }^{\phi}\right)
\end{aligned}
$$




$$
\begin{aligned}
& +\frac{\mathrm{e}}{\mathrm{m} \omega_{\mathrm{o}}^{2}} \frac{\mathrm{E}_{\mathrm{o}}}{\mathrm{k}_{\mathrm{o}}}\left(1-\alpha^{2}\right)^{1 / 2} \exp \left(i \mathrm{k}_{\mathrm{o}} \mathrm{z}\right)\left\{2 i \mathrm{k}_{\mathrm{oz}} \mathrm{k}_{\mathrm{ox}} \frac{\partial^{2} \phi_{-}}{\partial \mathrm{z}^{2}}\right. \\
& \left.+\frac{\partial \phi_{-}}{\partial z}\left[2 k_{x}\left(k_{o z}^{2}-k_{o x}^{2}\right)\right]+i \dot{\phi}_{-}\left(2 k_{x}^{2} k_{o x} k_{o z}\right)\right\} \\
& \left(\frac{3 v_{e}^{2}: \partial^{2}}{\omega^{2}} \frac{(z+\Delta z)}{\partial z^{2}}-\frac{2 \gamma}{\omega}\right)\left(\frac{\partial^{2}}{\partial z^{2}}-k_{\perp}^{2}\right) \phi_{-}-\frac{1}{L} \frac{\partial \phi_{-}}{\partial z} \\
& \because=\frac{\alpha i e k_{y}^{E} E_{o}}{m \omega_{o}^{2}} \exp \left(-i k_{o z} z\right)\left(-2 i k_{o z} \frac{\partial \phi}{\partial z}+2 k_{x} k_{n x} \phi\right) \\
& +\frac{e}{m \omega_{0}^{2}} \frac{E_{o}}{k_{o}}\left(1-\alpha^{2}\right)^{1 / 2} \exp \left(-i k_{o z} z\right)\left\{-2 i k_{o z} k_{o x} \frac{\partial^{2} \phi}{\partial z^{2}}\right. \\
& \left.\left.+\frac{\partial \phi}{\partial z}\left[2 k_{x}\left(k_{o x}^{2}-k_{o z}^{2}\right)\right]-i \phi\left(2 k_{x}^{2} k_{o x} k_{o z}\right)\right]\right\} \text {. }
\end{aligned}
$$

Here, $k_{o z}=k_{o} \sin \theta$ and $\Delta z \approx\left(3 v_{e}^{2} L / \omega^{2}\right)\left(k_{o x}^{2}-2 k_{x} k_{o x}\right)+(4 \delta L / \omega)$, $\omega=\omega_{0} / 2+\delta, \delta / \omega \ll 1$. $k_{0}$ has been neglected relative to $k_{\perp}$ in the constant terms of Eq. (2), since $k_{0} \ll k_{\perp} \sim 1 / \lambda_{D}$; however, the exponential mismatch term $\exp \left(\mathrm{ik}_{\mathrm{oz}} \mathrm{z}\right)$ has been retained.

We may solve Eq. (2) by fourier transforming, with $\phi(z)=$ $\int d s \exp (i s z) f(s)$ and $\phi_{-}(z)=\int d s \exp (i s z) f_{-}(s) \cdot$ Letting

$$
\begin{aligned}
& f(s)=\left(s^{2}+k_{\perp}^{2}\right)^{-1 / 2} \exp \left(i \lambda{ }_{D}^{2} s^{3} L\right) g(s) \\
& f_{-}(s)=:\left(s^{2}+k_{\perp}^{2}\right)^{-1 / 2} \exp \left(i \lambda_{D}^{2} s^{3} L\right) g_{-}(s),
\end{aligned}
$$

we obtain, after some algebra,

$$
\begin{aligned}
& \frac{\partial g\left(S+k_{o z} / 2\right)}{\partial S}+\frac{2 i \gamma L}{\omega} g\left(s+\frac{k_{O z}}{2}\right)=\frac{-L \gamma_{O} g_{-}\left(S-k_{O z} / 2\right)}{\left(S+k_{O z} / 2\right)^{2}+k_{\perp}^{2}} \\
& \text { - }\left[\alpha_{O}+\left(s-\frac{k_{O z}}{2}\right) \alpha_{1}-\left(s-\frac{k_{O z}}{2}\right)^{2} \alpha_{2}\right] \quad \exp \left[-i \lambda{ }_{D}^{2} L\left(3 k_{O z} s^{2}+\frac{k_{o z}^{3}}{4}\right)\right]
\end{aligned}
$$




$$
\begin{aligned}
& \frac{\partial g_{-}\left(S-k_{o z} / 2\right)}{\partial S}-\left(\frac{2 i \gamma L}{\omega}+i \Delta z\right) g_{-}\left(s-\frac{k_{o z}}{2}\right)=+\frac{L \gamma_{0} g\left(S+k_{o z} / 2\right)}{\left(S-k_{O z} / 2\right)^{2}+k_{\perp}^{2}} \\
& \text { - }\left[\alpha_{0}+\left(s+\frac{\mathrm{o}_{\mathrm{Oz}}}{2}\right) \alpha_{1}-\left(s+\frac{\mathrm{k} O z}{2}\right)^{2} \alpha_{2}\right] \exp \left[i \lambda_{D}^{2} L\left(3 k_{O z} s^{2}+\frac{k_{o z}^{3}}{4}\right)\right] \text {. }
\end{aligned}
$$

where $\gamma_{0}=2 e E_{o} / m_{0}^{2}, \alpha_{0}=+\alpha k_{y} k_{x} k_{o x}-\left(1-\alpha^{2}\right)^{1 / 2} k_{x}{ }^{2} k_{o x} k_{o z} / k_{0}$, $\alpha_{2}=-\left(1-\alpha^{2}\right)^{1 / 2} \mathrm{k}_{o z} \mathrm{k}_{o x} / \mathrm{k}_{\mathrm{o}}, \alpha_{1}=+\alpha \mathrm{k}_{\mathrm{y}} \mathrm{k}_{\mathrm{oz}}-\left(1-\alpha^{2}\right)^{1 / 2} \mathrm{k}_{\mathrm{x}}\left(\mathrm{k}_{\mathrm{oz}}^{2}-\mathrm{k}_{\mathrm{ox}}^{2}\right) / \mathrm{k}_{\mathrm{o}}$, and where we have let $s=s-k_{o z} / 2$. We have approximated $\left\{\left[\left(\mathrm{s}+\mathrm{k}_{\mathrm{oz}} / 2\right)^{2}+\mathrm{k}_{\perp}^{2}\right] /\left[\left(\mathrm{s}-\mathrm{k}_{\mathrm{oz}} / 2\right)^{2}+\mathrm{k}_{\perp}^{2}\right]\right\}^{1 / 2} \approx 1$. If we further let

$$
\begin{aligned}
& u(s)=g\left(s+\frac{k_{O z}}{2}\right) \exp \left(-\frac{3}{2} i k_{O z} L \lambda_{D}^{2} s^{2}-\frac{1}{8} i \lambda_{D}^{2} L k_{O z}^{3}\right) \\
& u_{-}(s)=g_{-}\left(s-\frac{k_{O z}}{2}\right) \exp \left(\frac{3}{2} i k_{O z} L \lambda_{D}^{2} s^{2}+\frac{1}{8} i \lambda_{D}^{2} L k_{O z}^{3}\right)
\end{aligned}
$$

We then obtain

$$
\begin{aligned}
& \frac{\partial u}{\partial s}+u\left(\frac{2 i \gamma L}{\omega}-3 i k_{o z} L \lambda_{D}^{2} S\right) \\
& =-\frac{\mathrm{L} \gamma_{\mathrm{O}} \mathrm{u}_{-}}{\left(\mathrm{s}+\mathrm{k}_{\mathrm{Oz}} / 2\right)^{2}+\mathrm{k}_{\perp}^{2}}\left(\alpha_{\circ}+\left(\mathrm{s}-\frac{\mathrm{k}_{\mathrm{Oz}}}{2}, \alpha_{1}-\left(\mathrm{s}-\frac{\mathrm{k}_{\mathrm{Oz}}}{2}\right)^{2} \alpha_{2}\right)\right. \\
& \frac{\partial u_{-}}{\partial S}-u_{-}\left(\frac{2 i \gamma L}{\omega}+i \Delta z-3 i k_{O z} L \lambda_{D}^{2} S\right) \\
& =+\frac{\mathrm{L} \gamma_{\mathrm{O}} \mathrm{u}}{\left(\mathrm{s}-\mathrm{k}_{\mathrm{Oz}} / 2\right)^{2}+\mathrm{k}_{1}^{2}}\left[\alpha_{\mathrm{O}}+\left(\mathrm{s}+\frac{\mathrm{k} \mathrm{Oz}}{2}\right) \alpha_{1}-\left(s+\frac{\mathrm{k} O z}{2}\right)^{2} \alpha_{2}\right]
\end{aligned}
$$

Equation (6a) and Eq. (6b) may be combined into a single second order equation for $u$ by taking the derivative of Equation $(6 a)$. If we then let $u(S)=W(S) \exp \left(-\int^{S} d \zeta h(\zeta) / 2\right)$, where 


$$
\begin{gathered}
\quad h(s)=-i \Delta z+\frac{2\left(s+k_{O z} / 2\right)}{\left(S+k_{O z} / 2\right)^{2}+k_{1}^{2}} \\
\therefore \quad-\frac{\alpha_{1}-2\left(s-k_{O z} / 2\right) \alpha_{2}}{\alpha_{0}+\left(S-k_{O z} / 2\right) \alpha_{1}-\left(S-k_{O z} / 2\right)^{2} \alpha_{2}},
\end{gathered}
$$

we can obtain, after much algebra,

$$
\frac{\partial^{2} W}{\partial S^{2}}+W\left(F_{0}+F_{1}+F_{2}\right)=0,
$$

where

$$
\begin{aligned}
& F_{0}=L^{2}\left\{\gamma_{0}^{2} \frac{\left[\alpha_{0}+S \alpha_{1}-\left(S^{2}-k_{O z}^{2} / 4\right) \alpha_{2}\right]^{2}-k_{O z}^{2}\left(\alpha_{1}^{2} / 4+\alpha_{2} \alpha_{0}\right)}{\left(S^{2}+k_{O z}^{2} / 4+k_{1}^{2}\right)^{2}-S^{2} k_{O Z}^{2}}\right. \\
& \left.\therefore \quad+\left(\frac{2 \gamma}{\omega}+\frac{\Delta z}{2 L}-3 k_{O Z} \lambda_{D}^{2} s\right)^{2}\right\} \\
& F_{1 .}=L\left\{-3 i k_{O z} \lambda_{D}^{2}+\left(\frac{2 \gamma i}{\omega}-3 i k_{o z} \lambda_{D}^{2} S+\frac{i \Delta z}{2}\right) \cdot\right. \\
& \left.\left[\frac{2\left(s+k_{o z} / 2\right)}{\left(s+k_{o z} / 2\right)^{2}+k_{l}^{2}}-\frac{\alpha_{1}-2\left(s-k_{o z} / 2\right) \alpha_{2}}{\alpha_{0}+\alpha_{1}\left(s-k_{o z} / 2\right)-\left(s-k_{o z}^{\prime} / 2\right)^{2} \alpha_{2}}\right]\right\} \\
& \mathrm{F}_{2}=-\frac{\mathrm{h}^{2}}{4}=\frac{1}{2} \frac{\partial \mathrm{h}}{\partial \mathrm{S}}-\frac{\Delta \mathrm{z}^{2}}{4}-\frac{i \Delta z}{2}\left[\frac{2\left(\mathrm{~s}+\mathrm{k}_{\mathrm{Oz}} / 2\right)}{\left(\mathrm{S}+\mathrm{k}_{\mathrm{Oz}} / 2\right)^{2}+\mathrm{k}_{\perp}^{2}}\right. \\
& \left.-\frac{\alpha_{1}-2\left(s-k_{o z} / 2\right) \alpha_{2}}{\alpha_{0}+\left(s-k_{o z} / 2\right) \alpha_{1}-\left(s-k_{o z} / 2\right)^{2} \alpha_{2}}\right]
\end{aligned}
$$

We now may solve Eq. (8), in a manner similar to that of T.i.u and Rosenbluth. ${ }^{3}$ We find $\Delta$ by assuming $L$ large and neglecting $F_{1}$ and $F_{2}$ in Eq. (8). $F_{1}$ is then included as a small perturbation that determines the correction to. $\Delta$. We may also simplify $F_{0}$ by reglecting $k_{0}^{2}$ relative to $k_{\perp}^{2}$. Temporal instability will first occur when $\left.F_{0}\right|_{\Delta, S}=\left.\left(\partial F_{0} / \partial S\right)\right|_{\Delta, S}$ $=0$ for some $\operatorname{Im} \Delta>0$, or equivalently when two turning points of $\mathrm{F}_{\mathrm{O}}$ coalesce for $\operatorname{Im} \Delta>0$. This is a necessary requirement for 
$W(S)$ to be bounded in the $S$ plane, which by the property of Fourier transforms bounds $\phi(z)$ in $z$. We note that

$$
\left.\frac{\partial F_{0}}{\partial S}\right|_{\Delta}=-\left.\left.\frac{\partial \Delta}{\partial S}\right|_{F_{0}} \frac{\partial F_{0}}{\partial \Delta}\right|_{S}
$$

Since $\left.\left(\partial \mathrm{F}_{0} / \partial \Delta\right)\right|_{S} \approx(4 / \omega)\left[(2 \gamma / \omega)+\Delta z / 2 L-3 k_{o z} \lambda_{D}^{2} S\right] \neq 0$ in general, we need only require $\left.F_{0}\right|_{\Delta, s}=\left.(\partial \Delta / \partial S)\right|_{F_{0}=0}=0$. We may first consider the limit of $k_{o z} \ll k_{o x}$. The equations for $\Delta$ become

$$
\begin{aligned}
\frac{2 \Delta}{\omega} & =-\frac{2 i \Gamma}{\omega}+3 k_{O z} \lambda_{D}^{2} s+i \gamma_{0} \frac{\alpha_{0}+s \alpha_{1}}{\left(S^{2}+k_{\perp}^{2}\right)}-\frac{\Delta z}{2 L} \\
0 & =3 k_{O z} \lambda_{D}^{2}\left(s^{2}+k_{\perp}^{2}\right)^{2}-i \gamma_{0} \alpha_{1}\left(s^{2}-k_{\perp}^{2}\right)-2 i s \gamma_{0} \alpha_{0},
\end{aligned}
$$

where $\alpha_{0}=+\alpha \mathrm{k}_{\mathrm{y}} \mathrm{k}_{\mathrm{x}} \mathrm{k}_{\mathrm{ox}}, \alpha_{1}=+\left(1-\alpha^{2}\right)^{1 / 2} \mathrm{k}_{\mathrm{x}} \mathrm{k}_{\mathrm{ox}}^{2} / \mathrm{k}_{\mathrm{o}}$.

We have chosen the + sign for the last term in Eq. (10a) for instability for $S>0$. We first let $\alpha_{0}=0(\alpha=0)$ and $\mathrm{k}_{\perp}=\mathrm{k}_{\mathrm{x}}$. For $\gamma_{0} \alpha_{1} /\left(3 \mathrm{k}_{\mathrm{oz}}{ }_{\mathrm{D}}^{2} \mathrm{k}_{\perp}^{2}\right) \gg \mathrm{l}, \mathrm{Eq} \cdot(10 \mathrm{~b})$ yields $\mathrm{s}^{2} \approx \mathrm{k}_{\perp}^{2}$. Equation (10a) then yields $\left(\mathrm{V}_{\mathrm{o}} \equiv \mathrm{eE_{ \textrm {o } }} / \mathrm{m} \omega_{0}\right)$

$$
\operatorname{Im} \dot{\Delta}=\Delta_{i}=\frac{\mathrm{k}_{\mathrm{Ox}} \mathrm{V}_{\mathrm{O}}}{4}-\Gamma \text {, }
$$

which results in the normal homogeneous plasma threshold $\mathrm{k}_{\mathrm{ox}} \mathrm{V}_{\mathrm{o}} / 4$ $=\dot{\Gamma}$. The other solution to Eq. (10b) when $\alpha_{0}=0$ is $\mathrm{s}^{2} \cong i \alpha_{1} \gamma_{0} / 3 \mathrm{k}_{\mathrm{oz}} \lambda_{\mathrm{D}}^{2}$, which yields $\Delta_{i}+\Gamma \approx \mathrm{k}_{\mathrm{o}} \mathrm{v}_{\mathrm{o}}\left(3 \mathrm{k}_{\mathrm{x}} \mathrm{k}_{\mathrm{oz}} \lambda_{\mathrm{D}}^{2} / 2 \mathrm{k}_{\mathrm{o}} \gamma_{\mathrm{o}}\right)^{1 / 2}$; we will ignore this solution since the root $s^{2} \approx k_{\perp}^{2}$ clearly gives the higher growth rate. A numerical analysis of Eq. shows that $\Delta_{i}+\Gamma>0$ for $\gamma_{0}\left(\alpha_{1} / 3 \mathrm{k}_{\mathrm{Oz}} \lambda_{\mathrm{D}}^{2} \mathrm{k}_{1}^{2}\right)>0.86$. This results in the condition for absolute instability:

$$
\theta<0.775 \frac{\mathrm{V}_{\mathrm{O}}}{\mathrm{c}} \frac{\mathrm{l}}{\mathrm{k}_{\mathrm{O}} \mathrm{k}_{\perp} \lambda_{\mathrm{D}}^{2}} .
$$


Equation (12) is valid only for $\theta \ll 1$ and $\theta>\left(3 / k_{0} L\right)^{1 / 3}$.

We may now find the first order in $1 / L$ corrections to $\Delta_{i}$ by letting $\Delta=\Delta_{1}+\Delta_{2},\left|\Delta_{2}\right| \ll\left|\Delta_{1}\right|$, and expanding. Eq. (7)

$$
\frac{\partial^{2} W}{\partial S^{2}}+W\left[\left.\frac{1}{2} \frac{\partial^{2} F_{0}}{\partial S^{2}}\right|_{S_{0}}\left(S-S_{0}\right)^{2}+\left.\frac{\partial F_{0}}{\partial \Delta}\right|_{S_{0}} \Delta_{2}+F_{1}\left(S_{O^{\prime}} \Delta_{I}^{\prime}\right)\right]=0 \text {. }
$$

This is simply the quantum harmonic oscillator problem, which has eigenvalues

$$
\Delta_{i}=-\Gamma+\frac{k_{0 x} V_{0}}{\Delta}-\frac{\left(n+\frac{1}{2}\right) \omega_{p}}{2 k_{\perp} \tau_{1}}
$$

For our large $L$ expansion to be valid, the last term in Eq. (14) must be a small correction to $\Delta_{i}$.

We may now apply this method of analysis to the problem of arbitrary angle of incidence. First, we consider $\alpha=0$. Our coefficients in Eq. (8) are then: $\alpha_{0}=-\mathrm{k}_{\mathrm{x}}^{2} \mathrm{k}_{\mathrm{ox}} \mathrm{k}_{\mathrm{oz}} / \mathrm{k}_{\mathrm{o}}$, $\alpha_{1}=-\mathrm{k}_{\mathrm{x}}\left(\mathrm{k}_{\mathrm{Oz} z}^{2}-\mathrm{k}_{\mathrm{ox}}^{2}\right) / \mathrm{k}_{\mathrm{o}}$ and $\alpha_{2}=-\mathrm{k}_{\mathrm{oz}} \mathrm{k}_{\mathrm{ox}} / \mathrm{k}_{\mathrm{o}}$. Letting $\mathrm{k}_{\mathrm{x}}=\mathrm{k}_{1}$, $\left(\gamma_{0} k_{0} / 3 k_{O z} k_{\perp} \lambda_{D}^{2}\right)>1$, and using the same analysis as before, we obtain after some algebra the growth rate (to lowest order in $1 / L)$

$$
\Delta_{i}=-\Gamma+\frac{k_{0} v_{0}}{4}
$$

For the case of $\alpha=1$, our coefficients are:

$\alpha_{0}=+k_{x}{ }_{x} k_{o x}, \alpha_{1}=k_{y} k_{o z}$, and $\alpha_{2}=0$. For $\left(\gamma_{0} k_{0} / 2 k_{o z} k_{\perp} \lambda_{D}^{2}\right)>1$, we obtain

$$
\Delta_{i}=-\Gamma+\frac{v_{o} k_{o}}{4} \frac{k_{y}^{k} x}{k_{i}^{2}}\left[\left(1+\frac{k_{y}^{2}}{k_{x}^{2}} \sin ^{2} \theta\right)^{1 / 2}+\cos \theta\right]_{(16)}
$$

It is easily shown that $\Delta_{i}+\Gamma$ is greatest for $k_{x}=k_{y} \cos \theta$. 
We then obtain

$$
\Delta_{i}=-\Gamma+\frac{k_{0} v_{o}}{4}
$$

The analysis has thus shown that absolute instability for the two plasmon parametric decay is possible in an infinite inhomogeneous plasma. It also states for the first time the requirement that

$$
\frac{V_{O}}{c}>k_{0} \cdot k_{\perp} \lambda \frac{2}{D} \sin \theta
$$

for absolute instability. For $\sin \theta=1$, Eq. (18) agrees with Liu and Rosenbluth. ${ }^{3}$ For $v_{0} / c$ much greater than threshold and for $\vec{E}_{0}$ either in the plane of incidence or perpendicular to the plane of incidence, the instability will grow with the homogeneous plasma growth rate.

Equation (18) results in a far lower threshold for absolute instability than previously stated in the literature. If we let $\mathrm{k}_{1} \sim 1 / \lambda_{\mathrm{D}}, \mathrm{Eq} \cdot(18)$ requires $\sin \theta<\left(\mathrm{V}_{\mathrm{O}} / \mathrm{c}\right)\left(\mathrm{k}_{\mathrm{O}} \lambda_{\mathrm{D}}\right)^{-1}$. For these values of $\theta$, we may estimate $\Delta_{2}$. It is easy to show that $\partial^{2} F_{0} / \partial S^{2} \sim\left(L^{2} k_{0}^{2} V_{0}^{2}\right) /\left(\omega^{2} k_{\perp}^{2}\right)$ and $\partial F_{0} / \partial \Delta \sim 2 k_{0} V_{0} L^{2} / \omega^{2}$. Equation (13) would then yield $\Delta_{2} \sim \omega_{\mathrm{p}} / \mathrm{k}_{\perp} \mathrm{L} \sim \mathrm{v}_{\mathrm{e}} / \mathrm{L}$. We must require $\left|\Delta_{2}\right| \ll\left|\Delta_{1}\right|$ for these solutions to be valid. This yields the condition $v_{o} / v_{e}>\left(k_{0} L\right)^{-1}$ for absolute instability. This is much lower than the $\left(V_{0} / v_{e}\right)^{2}>\left(k_{0} L\right)^{-1}$ threshold previously mentioned. 1,3 Making $\sin \theta<\left(v_{0} / c\right)\left(k_{0} \lambda_{D}\right)^{-1}$ is equivalent to letting $\sin \theta<\left(\mathrm{P} / 8 \mathrm{~T} \mathrm{e}^{\mathrm{nc}}\right)^{1 / 2}$. For a $10.6 \mu \mathrm{CO}_{2}$ laser emitting $10^{12} \mathrm{~W} / \mathrm{cm}^{2}$ on a $50 \mathrm{eV}$ quarter critical density plasma, this becomes $\theta<27^{\circ}$, a condition easily consistent with the WKR pump approximation fur $k_{0} L>1$. 
The main point of this work is the result that it will be easier to excite absolute instabilities for obliquely incident light waves than for normally incident radiation. This result could be of substantial importance in both the laser pellet and laser heated solenoid fusion schemes, in which the laser light should have ample opportunity to be obliquely incident on the density gradient at some point in the plasma.

\section{ACKNOWLEDGMENT}

The author is happy to acknowledge his valuable discussions with Drs. T. K. Chu, B. Cohen, C. S. Liu and R. White concerning this work.

This work was supported by U. S. Energy Research and Development Administration Contract $E(11-1)-3073$.

The author was supported by a National Science Foundation Graduate Fellowship.

\section{REFERENCES}

1 M. N. Rosenbluth, Phys. Rev. Lett. 29,565 (1972).

2 Y. C. Lcc and F. I. Kaw, Plyys. Rev. Lett. 32,135 (197.4).

3 C. S. Li.u and M. N. Rosenbluth, Phys. Fluids 19, 967 (1976).

4 T. Tang, Radio Science 5,111 (1970). 